Reprod. Nutr. Dévelop. 1980, 20 (2), 469-483.

\title{
Localisation, structure et activité des gènes ribosomiques dans le nucléole de l'ovocyte en prophase de méiose
}

\author{
par A. STAHL, C. MIRRE, Michèle HARTUNG, B. KNIBIEHLER \\ Laboratoire d'Histologie ef Embryologie II, \\ Faculté de Médecine \\ 27, boulevard Jean-Moulin, 13385 Marseille Cedex 4.
}

Summary. Localization, structure and activity of ribosomal genes in the oocyte nucleolus during meiotic prophase.

The structure, localization and activity of ribosomal genes were studied in quail, mouse and human oocytes. Meiotic prophase $I$ is a favourable stage for these studies since the connections between the nucleoli and the chromosomes are analyzable by light and electron microscopy.

In the newly-formed nucleolus at pachytene in the quail and mouse, the ribosomal genes were first localized in the fibrillar center, which was the nucleolar organizer at that stage. These genes were transcribed in the peripheral part of the fibrillar center. Superposition of the rDNA fibrils and the newly-synthesized rRNA led to the formation of a layer of electron-dense fibrils surrounding the fibrillar center.

The development of the nucleolus in the mouse oocyte at diplotene constitutes an excellent model for understanding the nucleolar organization. We compared the results of autoradiography, after $\mathrm{H} 3$-uridine incorporation, to those obtained by three-dimensional reconstruction of the nucleolus after serial sections, and found that the rDNA, initially compacted in the fibrillar center, had at least partially unravelled to become situated in the dense fibrillar strands of the nucleolonema. These strands, like the dense fibrillar layer surrounding the fibrillar center, were zones of rDNA transcription. Small fibrillar centers could be seen scattered throughout the nucleolonema; the number of these centers no longer corresponded to that of the nucleolar organizers. They were thus considered as areas of localized rDNA packing with no active transcription in their inner part.

The activity of the ribosomal genes in the human oocyte varied during the stages of meiotic prophase. rRNA synthesis, which was active in the oogonium, decreased considerably at zygotene and totally stopped at mid-pachytene ; it resumed at the end of pachytene and was very intense at diplotene. The temporary arrest of rRNA synthesis at pachytene was accompanied by segregation of the nucleolar components. The ribosomal genes gathered in the fibrillar center which was isolated from the other nucleolar components throughout pachytene. One outstanding feature of the human oocyte is that ribosomal genes originating from many acrocentric chromosomes were seen in juxtaposition in the same fibrillar center where they were wrapped in a silver-stained protein. The hypothesis is proposed that a proteolytic enzyme deficiency, perhaps related to advancing maternal age, would lead to non-disjunction of these genes. This mechanism could account for certain cases of trisomy, especially trisomy 21.

At diplotene, the resumption of rRNA synthesis, enhanced by amplification of the rDNA, led to the presence of numerous micronucleoli. 


\section{Introduction}

Les recherches effectuées chez les Vertébrés Inférieurs ont montré qu'un appareil très développé de synthèse protéique se constitue pendant l'ovogenèse. Composé essentiellement de ribosomes, il sera légué à l'embryon qui l'utilisera pour former les proféines nécessaires à la croissance et à la différenciation de ses tissus. Chez les Amphibiens, la synthèse d'ARN ribosomique s'arrête à la fin de la méiose et ne reprend qu'au début de la gastrulation. L'embryon dispose pour les premiers stades du développement d'une réserve de ribosomes formés pendant le stade diplotène de l'ovogenèse. Pendant ce stade, l'ovocyłe contient 600 à 2000 nucléoles qui sont le siège d'une synthèse active d'ARN ribosomique. Ils sont dus à l'amplification sélective des gènes ribosomiques, c'est-à-dire à une multiplication de ces gènes, qui a lieu au stade pachytène. L'amplification des gènes ribosomiques multiplie par 1000 la capacité de l'ovocyte à produire du rRNA.

Chez les Mammifères, et en particulier chez l'Homme, on ignore dans quelle mesure les matériaux contenus dans le cytoplasme de l'ovocyte seront utilisés pour les premiers stades du développement. Il est certain que des substances cytoplasmiques d'origine maternelle, formées pendant l'ovogenèse, sonł présentes dans l'œuf des Mammifères, mais on ne dispose que d'arguments indirects pour leur attribuer un rôle dans les premiers stades du développement.

Comparé aux cellules somatiques, l'œuf des Mammifères est une cellule énorme, puisque son diamètre varie de $60 \mu$ chez la Souris à $140 \mu$ chez l'Homme. La quantité totale d'ARN contenue dans l'œuf de Souris a été évaluée à 1,75 ng par Reamer (1963) et à 0,55 ng par Olds ef al. (1973). L'ovocyte du Lapin contiendrait, selon Manes (1969), 20 ng d'ARN, c'est-à-dire 35 fois plus que l'ovocyte de Souris. Comme l'ovocyte de Lapin a un volume 3 à 4 fois supérieur à celui de la Souris, il serait donc 10 fois plus riche en ARN. Toutefois, le contenu en ARN de l'ovocyte des Mammifères est très faible si on le compare à celui des Vertébrés Inférieurs, par exemple à celui du Xénope dont l'ovocyte mûr contient $5 \mu \mathrm{g}$ d'ARN.

En 1953 déjà, Flax avait montré par une technique histophotométrique que la teneur en ARN cytoplasmique de l'œuf de Souris augmentait linéairement pendant l'ovogenèse, puis diminuait entre l'ovulation et la fécondation. Les estimations les plus récentes sont basées sur les techniques autoradiographiques. Chez la Souris, l'incorporation d'uridine tritiée est relativement réduite dans l'ovocyte des petits follicules quiescents, mais elle augmente pendant la phase de croissance pour atteindre un maximum dans l'ovocyte des follicules qui comprennent 100 à 200 cellules folliculeuses (Oakberg, 1967 ; Moore et al., 1974). Dans le follicule cavitaire, la synthèse d'ARN diminuerait, elle serait presque nulle ou même absente dans les ovocytes qui approchent de la maturifé. De même, selon Baker et al. (1969), on ne pourrait plus détecter de synthèse d'ARN dans les ovocytes du Rat et du Singe parvenus au terme de leur croissance.

Toutefois, Wassarman et Letourneau (1976), en injectant directement l'uridine tritiée dans la cavité folliculaire, ont constaté que la synthèse de l'ARN se poursuit avec simplement une moindre intensité, jusqu'à la maturation méiotique, c'est-à-dire jusqu'à la disparition de la vésicule germinative. 
La plus grande partie de l'ARN de l'ovocyte est, comme pour les autres cellules, de l'ARN ribosomique. En première approximation, on peut admettre que l'œuf des Mammifères contient environ 100 fois plus d'ARN ribosomique qu'une cellule adulte.

Quand, où et comment cet ARN ribosomique est-il formé ? Nous avons tenté de répondre à ces questions en étudiant sa synthèse dans l'ovocyte de la Caille, de la Souris et dans l'ovocyte humain.

Nous nous sommes limités aux stades qui vont de l'ovogonie à l'ovocyte parvenu au diplotène (stade dictyotène pour la Souris). Il s'agit donc de stades embryonnaires ou fœtaux, étendus jusqu'à la période néonatale et postnatale pour la Souris et l'Homme.

\section{Matériel et méthodes}

Nous avons utilisé les techniques suivantes :

1) Techniques cytogénétiques ef cytochimiques en microscopie photonique. Parmi celles-ci, la technique à l'argent de Goodpasture et Bloom (1975) s'est révélée irès utile. Cetfe réaction colore des protéines acides associées à l'ARN ribosomique dans les zones de transcription active (Howell ef al., 1975 ; Howell, 1977 ; Schwarzacher et al., 1977, 1978).

2) Microscopie électronique et cytochimie ultrastructurale. Une mention particulière doit être faite pour la technique de Cogliati et Gautier (1973) basée sur l'emploi de l'ammine d'osmium, qui colore électivement le DNA.

3) Autoradiographie en microscopie photonique et électronique après incorporation d'uridine- ${ }^{3} \mathrm{H}$. Pour la microscopie électronique, la technique de Salpeter et Bachmann (1972) a été utilisée avec une durée d'exposition de 4 mois.

4) Hybridation in situ selon la technique de Gall et Pardue (1969) en utilisant de l'ARN $28 S$ et 185 marqué au tritium obtenu à partir de cellules HeLa. Les préparations recouvertes par une émulsion Kodak NTB2 ont été exposées pendant 3 mois.

5) Autoradiographie après incubation pendant $3 \mathrm{~h}$ dans un milieu contenant de l'actinomycine $\mathrm{D}$ tritiée $(100 \mu \mathrm{Ci} / \mathrm{ml})$. Durée de l'exposition : 4 mois.

\section{Observations et commentaires}

I. - La synthèse des ARN ribosomiques et la formation du nucléole dans l'ovocyfe de la Caille.

L'ovocyte de la Caille présente un exemple schématique de la distribution et du fonctionnement des gènes ribosomiques. En effet, au stade pachytène moyen, la transcription de ces gènes a lieu à l'extrémité de certains bivalents formés par l'appariement des microchromosomes porteurs d'un organisateur nucléolaire. L'ovocyte de la Caille montre une particularité morphologique assez rare : le bivalent nucléolaire pénètre dans le nucléole en voie de formation. Son extrémité active envoie des fibres de chromatine porteuses du rDNA à l'intérieur du centre fibrillaire du nucléole (fig. 1). Ainsi la disposition morphologique observée chez la Caille suggère déjà que c'est le centre fibrillaire du nucléole qui contient les gènes ribosomiques (Mirre et Stahl, 1976). 
Cefte hypothèse, qui avait déjà été avancée par Goessens (1973-1976) et par Jordan et Luck (1976) sur un matériel différent, a été confirmée par les arguments suivants : 1) Le centre fibrillaire contient un lacis de fibrilles de DNA qui peut être coloré électivement par la technique à l'amine d'osmium de Cogliati et Gautier (Mirre et Stahl, $1978 b)$; 2) La nature ribosomique de ce DNA est démontrée par la technique de l'hybridation in sifu (Knibiehler ef al., 1977).

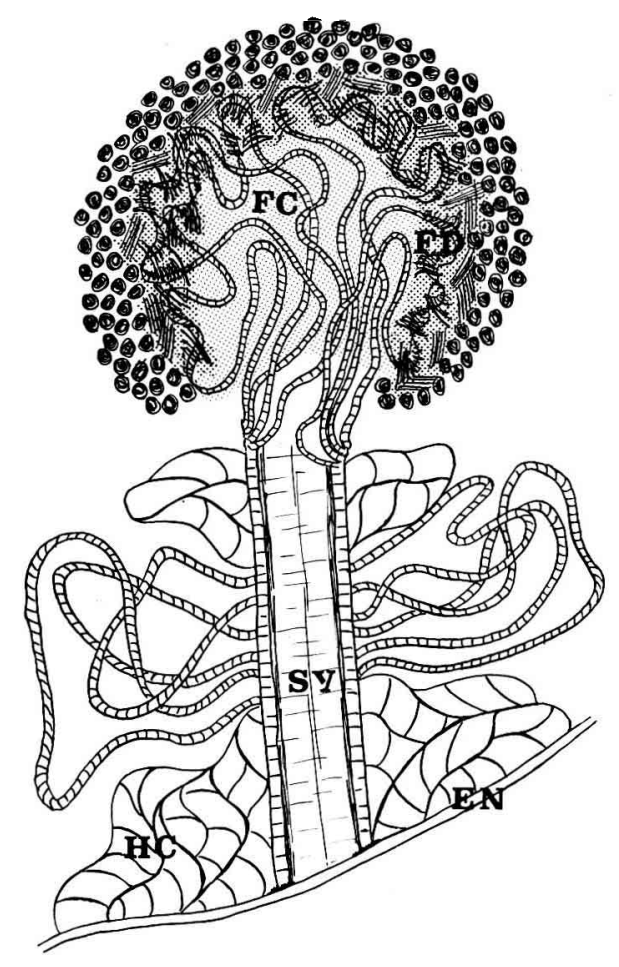

FIG. 1. - Représentation schématique du nucléole au début de sa formation au slade pachytène dans l'ovocyfe de Caille. Le nucléole néoformé est situé à l'extrémité d'un bivalent porteur de gènes ribosomiques appariés. Les fibres de chromatine qui contiennent le rDNA pénètrent dans le centre fibrillaire (FC) du nucléole. La transcription du rDNA a lieu dans la zone périphérique du centre fibrillaire. Elle entraîne la formation d'une couche de fibrilles denses (FD), où se superposent le rDNA et le rRNA nouvellement formé. Le reste du nucléole présente une constitution fibrillogranulaire. SY : complexe synaptonémal. HC : hétérochromatine. EN : enveloppe nucléaire.

L'étude de la transcription du rDNA contenu dans le centre fibrillaire a été réalisée par autoradiographie après incorporation d'uridine- ${ }^{3} \mathrm{H}$. On constate que le rDNA n'est transcrit que dans la zone périphérique du centre fibrillaire. La superposition du rDNA et du rRNA nouvellement formé auquel se fixent très rapidement des protéines se traduit par l'apparition, autour du centre fibrillaire, d'une couche de fibrilles denses (Mirre et Stahl, 1978a, b). Ainsi s'explique le fait que le centre fibrillaire est, quel que soit le nucléole étudié, pratiquement toujours entouré par une couche fibrillaire opaque aux électrons, qui n'est pas autre chose que la zone de transcription du rDNA.

La présence de DNA dans la couche de fibrilles denses qui entoure le centre fibril- 
laire a pu être démontrée en faisant incuber l'ovocyte en présence d'actinomycine D tritiée. Cette drogue provoque la séparation des constituants nucléolaires en même temps qu'elle se fixe sur le DNA. La localisation du DNA est révélée par autoradiographie. Nous avons ainsi constaté que le DNA nucléolaire est situé non seulement dans le centre fibrillaire, mais également dans sa couche enveloppante de fibrilles denses.

La figure 1 résume de façon schématique l'ensemble de ces résultats.

II. - La synthèse des ARN ribosomiques ef la formation du nucléole dans l'ovocyfe de la Souris.

1. - La formation d'un nouveau nucléole. - Chez la Souris on assiste, comme chez la Caille, à une synthèse d'ARN ribosomique au stade pachytène moyen, qui se traduit par la formation de nouveaux nucléoles.

Les bivalents porteurs de gènes ribosomiques appariés sont insérés sur l'enveloppe nucléaire par l'extrémité qui est entourée d'hétérochromatine centromérique. On sait que chez la Souris, les chromosomes porteurs d'un organisateur nucléolaire présentent une constriction secondaire qui est située juste après la région centromérique, elle-même en position terminale (Ford, 1966 ; Dev ef al., 1971 ; Eicher, 1972). La présence de rDNA dans la constriction secondaire a été vérifiée par hybridation in situ dans les cellules somatiques (Elsevier et Ruddle, 1975 ; Henderson ef al., 1976) et dans les cellules germinales (Stahl ef al., 1977, 1978).

Le nucléole néoformé apparaît en regard de la région du bivalent qui correspond à la constriction secondaire. Il présente au début toujours le même aspect, étant constitué d'un centre fibrillaire presque complètement enveloppé par une couche de fibrilles opaques aux électrons. Cette couche ne manque que dans une zone qui fait face au bivalent; dans cette zone on assiste à la pénétration dans le centre fibrillaire de fibres de chromatine émanées de la région de la constriction secondaire du bivalent. Cette disposition est fondamentalement identique à celle observée chez la Caille, à cette différence près que le nucléole se forme sur le côté et non à l'extrémité du chromosome nucléolaire (fig. 2a).

En fait ce sont deux nucléoles qui sont formés en regard de la région de la constricfion secondaire car les deux organisateurs nucléolaires appariés fonctionnent de façon synchrone et symétrique.

L'hybridation rRNA/rDNA in situ montre que le centre fibrillaire contient effectivement, à ce stade, les gènes ribosomiques (Stahl ef al., 1977, 1978).

L'étude autoradiographique du site de transcription confirme que celle-ci ne s'effectue pas en n'importe quelle zone du centre fibrillaire. Après incorporation d'uridine $-{ }^{3} \mathrm{H}$, le marquage est toujours limité à la couche enveloppante de fibrilles denses. Les unifés de transcription sont par conséquent situées dans la zone périphérique du centre fibrillaire (Mirre et Stahl, 1978a, b).

2. - Le développement du nucléole. - A la fin du stade pachytène, les deux nucléoles formés par les 2 organisateurs nucléolaires appariés, fusionnent. A partir de ce moment, le nucléole néoformé contient deux centres fibrillaires, émanés chacun de l'organisateur nucléolaire correspondant. On constate que la couche enveloppante de fibrilles denses s'est étendue sous la forme d'une bande, d'une plage ou de cordons qui 
sont formés uniquement de fibrilles opaques aux électrons. Dans leur région distale, des granules apparaissent, de sorte que la région du nucléole siłuée le plus loin du centre fibrillaire a un aspect fibrillo-granulaire. Cette région montre un aspect réticulaire car les cordons fibrillo-granulaires sont anastomosés les uns avec les autres (fig. $2 b$ et $c$ ).

Au début du stade diplotène, l'ensemble du nucléole a pris un aspect réticulaire en même temps que son volume s'est considérablement accru. Les cordons fibrillogranulaires forment dans leur ensemble anastomotique un aspect typique de nucléolonema (fig. 2d).

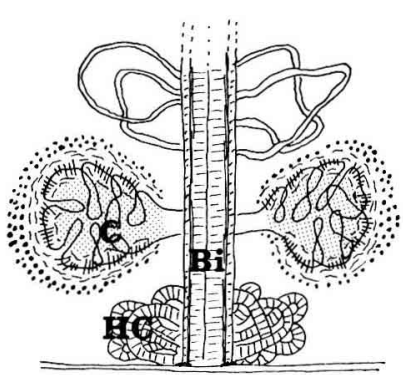

a

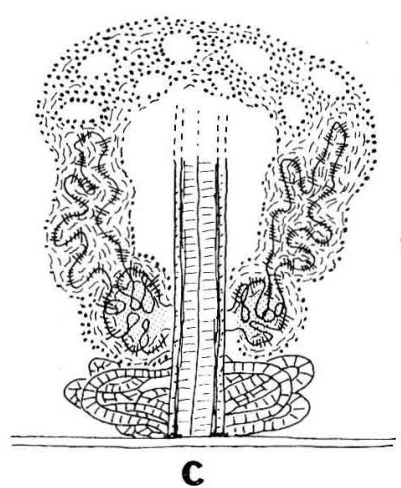

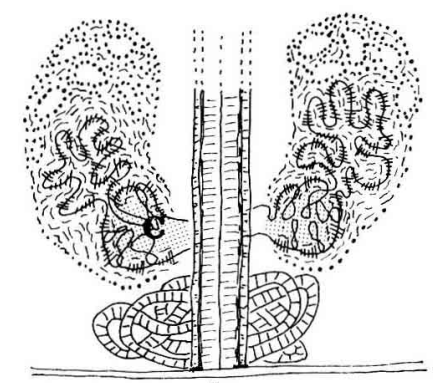

b

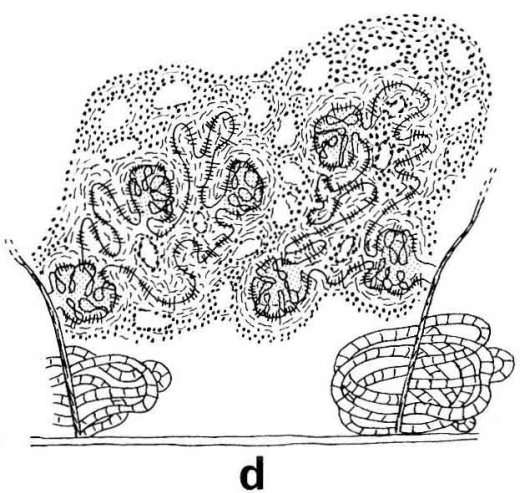

FIG. 2. - Formation du nucléole et répartition du rDNA dans l'ovocyte de Souris. a) Stade pachytène moyen. Deux nucléoles sont formés de façon synchrone en regard de la région de la constriction secondaire d'un bivalent $(\mathrm{Bi})$ porteur de gènes ribosomiques appariés. Les nucléoles néoformés montrent un centre fibrillaire (c) entouré par une couche de fibrilles denses et au-delà de cette dernière, le constituant fibrillo-granulaire. Les fibres de chromatine porteuses du rDNA pénètrent dans le centre fibrillaire. La transcription du rDNA s'effectue dans la couche de fibrilles denses localisée à la périphérie du centre fibrillaire. HC : hétérochromatine. b) Stade pachytène plus avancé. Les deux nucléoles augmentent de volume. Le rDNA, compacté au stade précédent dans le centre fibrillaire, se déroule et s'étend dans toute la partie du nucléole formé de fibrilles denses, où il est transcrit. L'extrémité distale du nucléole, fibrillogranulaire ou granulaire, prend un aspect réticulé. c) Stade pachyłène tardif : les deux nucléoles fusionnent. A ce stade, on ne voit encore que deux centres fibrillaires dans leur situation initiale. d) Stade diplotène précoce. Les deux chromosomes se séparent. Dans le nucléole réticulé on observe $: 1$ ) des cordons ce fibrilles denses, 2) de nombreux centres fibrillaires de petite taille. Le rDNA est transcrit à la périphérie des centres fibrillaires et dans les cordons fibrillaires denses. Les centres fibrillaires correspondent à des régions du nucléolonema où des portions de rDNA sont compactées et non transcrites. 
Un fait très caractéristique est l'apparifion de nombreux centres fibrillaires, dont le diamètre est nettement plus petit qu'au stade pachytène. Chacun de ces centres fibrillaires est toujours entouré, soit complètement soit incomplètement, par une couche de fibrilles denses.

Comme la Souris possède 5 paires de chromosomes porteurs de gènes ribosomiques, on est donc amené à se demander si chacun des nombreux centres fibrillaires néoformés peut encore être assimilé à un organisateur nucléolaire. Pour que cette proposition soit exacte, le nombre des centres fibrillaires ne devrait pas excéder 20 (l'ovocyte au diplotène contient 4C DNA).

Pour tenter de résoudre ce problème, nous avons fait des coupes en série de plusieurs noyaux d'ovocytes au stade diplotène et nous avons reconstruit dans les 3 dimensions chacun des nucléoles, en reportant de façon rigoureuse, le nombre et la position des centres fibrillaires. Il est apparu ainsi que le nombre total des centres fibrillaires de l'ensemble des nucléoles d'un noyau étaif de l'ordre d'une centaine, nombre qui n'a plus rien de commun avec celui des organisateurs nucléolaires.

L'analyse morphologique a par ailleurs montré que chaque centre fibrillaire se présentait comme une petite dilatation localisée du nucléolonema. La couche des fibrilles denses qui l'entoure est en effet toujours en continuité avec les cordons de même aspect du nucléolonema.

Enfin, nous avons suivi l'évolution de la transcription du rDNA du stade pachytène au stade diplotène, à toutes les étapes des transformations morphologiques que présente le nucléole en développement. D'abord limitée à la couche enveloppante des fibrilles denses, la zone de transcription s'étend progressivement à foutes les parties du nucléole formées de cordons fibrillaires denses. C'est ainsi que dans le nucléole réticulé du stade diplotène, la transcription s'observe : 1) aułour de chaque centre fibrillaire ; 2) dans l'ensemble des cordons fibrillaires denses du nucléolonema. Ce fait ne peut s'interpréter qu'en admettant que le rDNA, initialement compacté dans le centre fibrillaire, s'est déroulé et occupe maintenant l'ensemble du nucléolonema. Dès lors, les nombreux petits centres fibrillaires qui parsèment ce dernier doivent être considérés comme des zones de réserve contenant du rDNA encore localement compacté, mais pouvant éventuellement se dérouler ef devenir actif si les besoins en rRNA de la cellule viennent à s'accroître (Mirre ef Stahl, en préparation).

L'analyse des étapes de la formation du nucléole jointe à l'étude de la transcription du rDNA dans l'ovocyte de Souris a ainsi permis de comprendre l'organisation spatiale des gènes nucléolaires ef $a$ fourni les bases d'un modèle général de la constitution du nucléole, valable pour tous les types cellulaires pourvus d'un nucléole réticulé (c'est-à-dire à nucléolonema apparent). Cette analyse n'a été possible que parce que les relations entre le nucléole et les chromosomes porteurs de gènes ribosomiques restent visibles pendant une longue phase de la méiose où ces gènes sont activement transcrits. Le système que nous avons éfudié présente ainsi un avantage considérable sur les cellules somatiques habituellement utilisées pour les recherches nucléolaires.

III. - Synthèse des ARN ribosomiques ef évolution des nucléoles dans l'ovocyfe humain.

1. - La synthèse du rRNA. - L'activité des gènes ribosomiques a été étudiée par autoradiographie quantitative après incorporation d'uridine- ${ }^{3} \mathrm{H}$ depuis le stade d'ovogonie jusqu'au stade diplotène. 
Nous avons constaté que la transcription du rDNA est très active dans l'ovogonie, active également au stade leptotène. Elle décroît ensuite au zygotène pour devenir nulle au pachytène. La transcription reprend à la fin du pachytène pour afteindre au diplotène un niveau très élevé (Hartung et Stahl, 1978) (fig. 3).

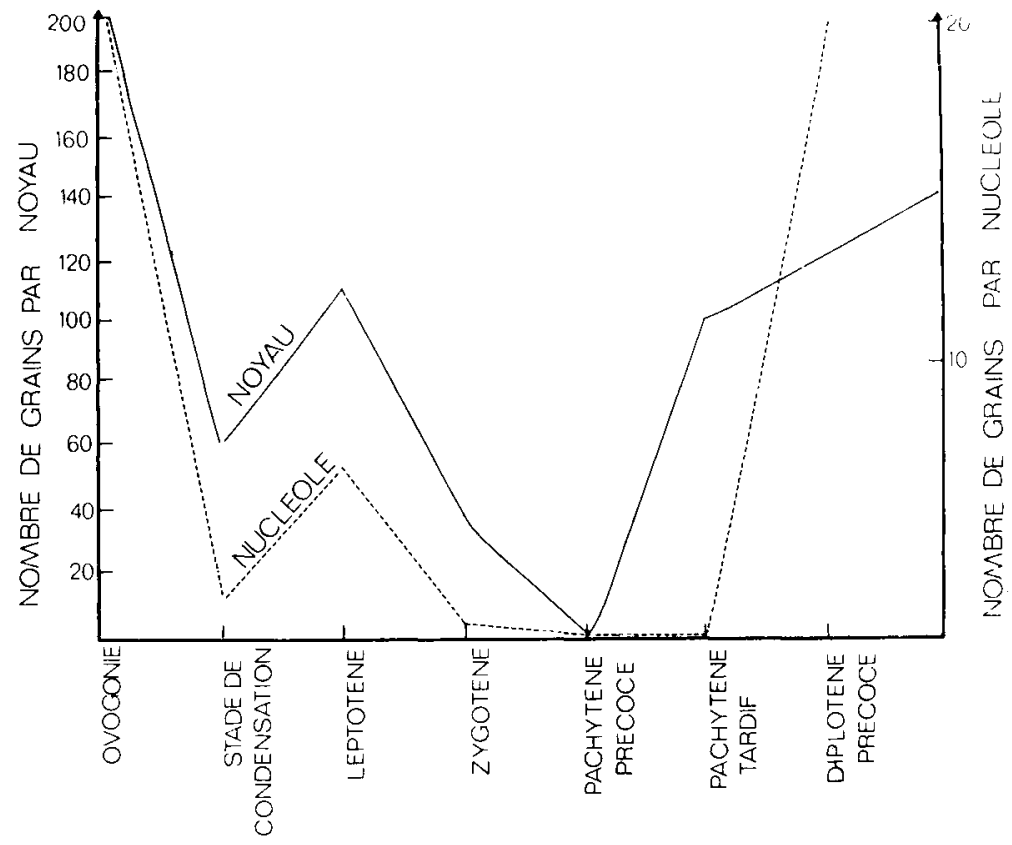

FIG. 3. - Variations de l'incorporation d'uridine- ${ }^{3} \mathrm{H}$ dans le nucléole et le noyau de l'ovocyte humain en prophose de méiose.

2. - Evolution morphologique du nucléole. - Les aspects observés en microscopie électronique ont été comparés avec ceux révélés par la technique à l'argent pour les organisateurs nucléolaires.

FIG. 4. - Ovocyte humain au stade pachyłène (détail). Après coloration par l'argent, le nucléole présente une zone fortement réactive juxtaposée à une zone non colorée. Un bivalent (Bi) est au contact de la zone colorée par l'argent. $\times 2400$.

FIG. 5. - Ovocyte humain au stade pochytène. Le nucléole montre une ségrégation spentanée de ses constituants. Le centre fibrillaire (CF) est pénétré par des fibres de chromatine émanées de la région de la constriction secondaire du bivalent $(\mathrm{Bi})$. EN : enveloppe nucléaire. $\times 17500$.

FIG. 6. - Stade diplotène précoce. Une couche de fibrilles denses réapparaît autour du centre fibrillaire (CF). Y. 20000.

FIG. 7. - Ovocyfe humain au stode diplotène avancé après coloration par la réaction à l'argent. Une réaction positive est observée sur le bord de plusieurs micronucléoles. NU : nucléole principal. $\times 2600$. 
L'ovogonie présente plusieurs nucléoles de type réticulé, pourvus de nombreux centres fibrillaires. La technique à l'argent colore des structures arrondies dont la taille et la distribution correspondent à celles des centres fibrillaires (Hartung, Mirre et Stahl, 1979).

Au stade leptotène, les centres fibrillaires du nucléole occupent une situation marginale. Les chromosomes sont visibles, sous la forme d'axes simples entourés de fibres de chromatine. On constate que des fibres de chromatine, émanées de l'extrémité de chromosomes acrocentriques, pénètrent dans le centre fibrillaire du nucléole. On retrouve ainsi dans l'ovocyle humain les relations caractéristiques entre le centre fibrillaire et les fibres de chromatine contenant le rDNA.

La technique à l'argent colore des zones arrondies situées au bord du nucléole, dont la topographie et les dimensions correspondent à celles des centres fibrillaires.

Au stade pachytène, après coloration par la technique à l'argent, le nucléole est divisé en deux zones dont l'une, de forme arrondie, est colorée en noir. Lorsque les relations d'un chromosome $D$ ou $G$ avec le nucléole ont été conservées, on constate que son extrémité aboutif précisément à la zone colorée par l'argent (fig. 4).

La microscopie électronique révèle à ce stade des faits d'un grand intérêt :

1) le nucléole est le siège d'une ségrégation spontanée de ses constituants : le centre fibrillaire est séparé du constituant fibrillo-granulaire (fig. 5 et 8). Cette ségrégation naturelle est en accord avec l'absence d'incorporation d'uridine trifiée, c'est-à-dire l'inactivité des cistrons ribosomiques. Cet arrêt de la transcription du rDNA est une particularité de l'ovocyte humain. Il ne s'observe pas chez la Souris et la Caille. Par contre, le même phénomène a été décrit par Tres (1975) dans le spermatocyłe humain. 2) Le centre fibrillaire du nucléole est pénétré par des fibres de chromatine émanées de la région de la constriction secondaire (ou tige) d'un ou plusieurs bivalents du groupe D ou $G$ (fig. 5 et 8 ).

3) II est évident que le centre fibrillaire correspond à la région qui est colorée par l'argent : la distribution spatiale est la même ainsi que les relations avec les bivalents nucléolaires. Cette conclusion a été confirmée sur un matériel différent par HernandezVerdun et al. (1978).

4) Lorsque le centre fibrillaire reçoit les fibres de chromatine émanées de 2 bivalents, son volume est le double de celui d'un centre fibrillaire qui ne reçoit que les fibres d'un bivalent. Si l'on tient compte du fait que chaque bivalent est formé de deux chromosomes appariés, et que chaque chromosome contient en fait deux chromatides, un tel centre fibrillaire contient les gènes nucléolaires juxtaposés ou peut-être même intriqués de 8 chromatides.

Cette situation pourrait constituer la base pathogénique des processus de nondisjonction des chromosomes acrocentriques, en particulier du 21, qui peuvent avoir pour conséquence une trisomie 21 . On sait en effet que les deux tiers des non-disjonctions dans la trisomie 21 se produisent à la 1 re division méiotique de l'ovocyte (Mattei et al., 1979). Déjà Ferguson-Smith ef Handmaker (1961) ef Ohno ef al. (1961) avaient suggéré que l'intrication des fibres de rDNA pourrait être une cause majeure de nondisjonction ef de translocation des chromosomes acrocentriques. On ignore tou! du rôle éventuel joué par les protéines colorées par l'argent mais un rapprochen ent peut être suggéré par le fait que dans les associations des chromosomes acrocen- 
reprend à la fin du stade pachytène. Ainsi se trouve confirmée notre interprétation selon laquelle la couche enveloppante de fibrilles denses est la zone de transcription (fig. 6 et 8 ).

Au début du stade diplotène, les nucléoles augmentent de volume ef leur partie centrale prend un aspect réticulé. Cependant, les centres fibrillaires restent pendant longtemps à la périphérie du nucléole, ce qui est attesté aussi bien par la réaction à l'argent que par la microscopie électronique.

Chez le fœus âgé de 6 à 8 mois, on assiste à la formation de nombreux micronucléoles. Ils apparaissent dans les régions du noyau qui n'ont aucune relation avec la constriction secondaire des chromosomes acrocentriques. lis sont par conséquent formés en dehors des organisateurs nucléolaires classiques. Nous avions émis l'hypothèse que ces micronucléoles étaient le résultat d'une amplification des gènes ribosomiques, par analogie avec les faits connus chez les Amphibiens (Stahl ef al., 1975). L'exactifude de cetfe opinion a été démontrẻe par Wolgemuth-Jarashow ef al. (1977) et Wolgemuth ef al. (1979) qui, en utilisant les techniques d'hybridation in situ, ont décelé du rDNA dans la plupart des micronucléoles. Nous avons pu récemment confirmer l'existence d'une amplification du rDNA dans l'ovocyte humain par la technique à l'argent. En effet, certains micronucléoles montrent sur l'un de leurs bords, une zone forlement colorée par l'argent qui correspond à une séquence de rDNA activement transcrite (fig. 7) (Hartung, Mirre et Stahl, 1979).

La signification biologique de cette amplification est obscure. Chez le Xénope, l'amplification est indispensable, sinon l'ovocyte mettrait plusieurs centaines d'années pour synthétiser le RNA ribosomique qu'il accumule ef qui sera nécessaire pour les premiers stades du développement, car les gènes nucléolaires ne redeviennent actifs qu'à partir de la gastrulation. Mais chez la Souris, la synthèse du rRNA reprend précocement : au stade de 2 blastomères selon Knowland et Graham (1972) et selon Hansman (1978) ou au stade 4 blastomères selon Moore (1975). Chez la Femme l'ovocyte reste quiescent, bloqué au stade diplotène pendant au moins une dizaine d'années et on ignore tout de l'activité des gènes ribosomiques aux premiers stades du développement. Il est possible que chez les Mammifères l'amplification - dans de modestes proportions - du rDNA ne soit pas due à la nécessité de stocker du rRNA pour le début de l'embryogenèse et que ce soit un excès inutile dû à une pression sélective insuffisante, comme Kidder (1976) l'avait suggéré pour l'ovocyte des Mollusques.

\section{Conclusions}

L'étude de la localisation, de la structure et de l'activité des gènes ribosomiques dans l'ovocyte en prophase de méiose permet de dégager les conclusions suivantes :

1) Dans l'ovocyte humain, l'activité des gènes ribosomiques varie au cours des stades de la prophase méiotique. La synthèse du rRNA, active dans l'ovogonie, diminue considérablement au zygotène et est totalement suspendue au pachytène moyen. Elle reprend à la fin du pachyłène et devient très intense au diplotène. Cet arrêt temporaire des synthèses ribonucléiques se produit pendant une phase de la mélose relativement courte où la plupart des auteurs situent les phénomènes de recombinaison, dont la survenue paraît attestée par une synthèse résiduelle de DNA (Stern et Hotta, 1974). 
L'arrêt de la synthèse du rRNA se traduit morphologiquement par la ségrégation des constituants du nucléole. Les gènes ribosomiques sont rassemblés dans le centre fibrillaire, isolé des autres constituants nucléolaires pendant la durée du stade pachytène. Une particularité de l'ovocyłe humain est la juxtaposition fréquente de gènes ribosomiques provenant de nombreux chromosomes acrocentriques dans un même centre fibrillaire, où ils sont englobés dans une protéine argyrophile. On ne peut exclure l'hypothèse que l'absence ou le déficit d'une enzyme protéolytique, en relation peut-être avec l'âge avancé de la mère, pourrait entraîner une malségrégation de ces gènes, expliquant ainsi certaines trisomies, en particulier du chromosome 21.

La reprise de la synthèse du RNA ribosomique au diplotène est renforcée par une amplification du rDNA qui a pour conséquence l'apparition de nombreux micronucléoles.

2) Dans le nucléole néoformé au pachytène chez la Caille ef chez la Souris, les gènes ribosomiques sont au débuf localisés dans le centre fibrillaire. La transcription de ces gènes a lieu dans la zone périphérique du centre fibrillaire. Elle se traduit par l'apparition d'une couche enveloppante de fibrilles denses.

3) L'évolution du nucléole au stade diplotène de l'ovocyte de Souris fournit un excellent modèle pour la compréhension de l'organisation nucléolaire. Les techniques d'analyse utilisées montrent que le rDNA, d'abord compacté dans le centre fibrillaire, se déroule au moins en partie pour se situer dans les cordons fibrillaires du nucléolonema qui sont, comme la couche fibrillaire enveloppante du centre fibrillaire, des zones de transcription du rDNA. Le nucléolonema montre de place en place de petits centres fibrillaires dont le nombre ne coïncide plus avec celui des organisateurs nucléolaires, et qui doivent être considérés comme des zones de compactage localisé du rDNA, sans transcription active dans leur partie interne.

Présenté au Colloque D. G. R. S. T. de Port Bail, 27 février-1er mars 1979.

Accepté en octobre 1979.

Remerciements. - Travail réalisé avec l'aide de la DGRST (Contrat No 77.7.1925) et du CNRS (ERA No 397).

\section{Références}

BAKER T. G., BEAUMONT H. M., FRANCHI L. L. 1969. The uptake of tritiated uridine and phenylalanine by the ovaries of rats and monkeys. J. Cell Sci., 4, 655-675.

COGLIATI R., GAUTIER A., 1973. Mise en évidence de l'ADN et des polysaccharides à l'aide d'un nouveau réactif « de type Schiff ». C. R. Acad. Sci. Paris, sér. D, 276, 3041-3044.

DEV V. G., GREWAL M. S., MILLER D. A., KOVRI R. E., HUTTON J. J., MILLER O. J., 1971. The quinacrine fluorescence karyotype of Mus musculus and demonstration of strain differences in secondary constrictions. Cylogenetics, 10, 436-451.

EICHER E. M., 1972. The identification of the chromosome bearing linkage group $X \mid 1$ in the mouse. Genetics, 69, 267-271.

ELSEVIER S. M., RUDDLE F. H., 1975. Location of genes coding for 185 and 28 S ribosomal RNA within the genome of Mus musculus. Chromosoma (Berl.), 52, 219-228.

FERGUSON-SMITH M. A., HANDMAKER S. D., 1961. Observation on the satellited human chromosomes. Lancet, 1, 638. 
FLAX M. H., 1953. Ribose nucleic acid and protein during oogenesis and early embryonic development in the mouse. Ph. D. Thesis, Columbia Univ.

FORD C. E., 1966. The use of chromosome markers, 197-206. In MICKLE H. S. LOUTIT, J. F. Tissue grafting and radiation. Acad. Press, New York.

GALL J. G., PARDUE M. L., 1969. Formation and detection of RNA-DNA hybrid molecules in cytological preparations. Proc. nat. Acad. Sci. (USA), 63, 378-383.

GOESSENS G., 1973. Les « centres fibrillaires » des nucléoles de cellules tumorales d'Ehrlich. C. $R$. Acad. Sci. Paris, sér. D, 277, 325-327.

GOESSENS G., 1974. Etude autoradiographique au microscope électronique des nucléoles de cellules tumorales d'Ehrlich. Mise en évidence d'ADN au niveau des centres fibrillaires. C. $R$. Acad. Sci. Paris, sér. D, 279, 991-993.

GOESSENS G., 1975. Etude ultrastructurale des nucléoles au cours du cycle cellulaire. Th. Doct. Univ. de Liège.

GOESSENS G., 1976. High resolution autoradiographic studies of Erhlich fumor cell nucleoli. Nucleolar labelling after ${ }^{3} \mathrm{H}$-actinomycin $\mathrm{D}$ binding to DNA or after ${ }^{3} \mathrm{H}$-thymidine or ${ }^{3} \mathrm{H}$-uridine incorporation in nucleic acids. Exp. Cell Res., 100, 88-94.

GOESSENS G. 1976. The nucleolar fibrillar centres in various cell types in vivo or in vitro. Cell Tiss. Res., 173, 315-324.

GOODPASTURE G., BLOOM S. E., 1975. Visualization of nucleolar organizer regions in Mammalian chromosomes using silver staining. Chromosoma, 53, 37-50.

HANSMAN I., GEBAUER L., BIHL L., GRIMM T., 1978. Onset of nucleolus organizer activity in early mouse embryogenesis and evidence for its regulation. Exp. Cell Res., 114, 263-268.

HARTUNG M., STAHL A., 1978. Autoradiographic study of RNA synthesis during meiotic prophase in the human oocyte. Cytogenef. Cell Genet., 20, 51-58.

HARTUNG M., MIRRE C., STAHL A., 1979. Nucleolar organizers in human oocytes at meiotic prophase I studied by the silver-NOR method and electron microscopy. Hum. Gen., 52, 295-308.

HENDERSON A. S., EICHER E. M., YU M. T., ATWOOD K. C., 1976. Variation in ribosomal RNA gene number in mouse chromosomes. Cytogenet. Cell Genet., 17, 307-316.

HENDERSON A. S., WARBURTON D., ATWOOD K. C., 1973. Ribosomal DNA connectives between human acrocentric chromosomes. Nature (London), 245, 95-97.

HERNANDEZ-VERDUN D., HUBERT J., BOURGEOIS C., BOUTEILLE M., 1978. Identification ultrastructurale de l'organisateur nucléolaire par la technique à l'argent. C. R. Acad. Sci. Paris, sér. $D, 287,1421-1423$.

HOWELL M. K., 1977. Visualization of ribosomal gene activity : silver stain protein associated with rRNA transcribed from oocyte chromosomes. Chromosoma, 62, 361-367.

HOWELL W. M., DENTON T. E., DIAMOND J. R., 1975. Differential staining of the satellite regions of human acrocentric chromosomes. Experientia, 31, 260-262.

JORDAN E. G., LUCK B. T., 1976. The nucleolus organizer and the synaptonemal complex in Endymion non scriptus (L.). J. Cell Sci., 22, 75-86.

KIDDER G. M., 1976. The ribosomal RNA cistrons in clam gametes. Develop. Biol., 49, 132-142.

KNIBIEHLER B., NAVARRO A., MIRRE C., STAHL A., 1977. Localization of ribosomal cistrons in the quail oocyte during meiotic prophase I. Exp. Cell Res., 110, 153-157.

KNOWLAND J., GRAHAM C., 1972. RNA synthesis at the two-cell stage of mouse development. J. Embryol. exp. Morph., 34, 291.

MANES C., 1969. Nucleic acid synthesis in preimplantation rabbit embryos. I. Quantitative aspects, relationship to early morphogenesis and prolein synthesis. J. exp. Zool. 172, 303-310.

MATTEI, J. F., GIRAUD, F. 1975,. Etude chromosomique des parents d'enfants trisomiques 21. Génét. hum., 23, 31-41.

MATTEI J. F., MATTEI M. G., AYME S., GIRAUD F., 1979. Origin of the extra chromosome in trisomy 21. Hum. Genet., 46, 107-110.

MIRRE C., STAHL A., 1976. Ultrastructural study of nucleolar organizers in the Quail oocyte during meiotic prophase I. J. Ultrastruct. Res., 56, 186-201.

MIRRE C., STAHL A., 19780. Ulitastructure and activity of the nucleolar organizer in the mouse oocyte during meiotic prophase. J. Cell Sci., 31, 79-100.

MIRRE C., STAHL A., 1978b. Peripheral RNA synthessis of fibrillar center in nucleoli of japanese quail oocyte and somatic cells. J. Ultrastruct. Res., 64, 377-387. 
MOORE G. P. M., 1975. The RNA polymerase activity of the preimplantation mouse embryo. J, Embryol. exp. Morph., 34, 291-298.

MOORE G. P. M., LINTERN-MOORE S., PETERS H., FABER M., 1974. RNA synthesis in the mouse oocyte. J. Cell Biol., 60, 416-422.

OAKBERG J. F., 1967. ${ }^{3} \mathrm{H}$-Uridine labeling in mouse oocytes. Arch. Anat. micr. Morph. Exp., 56, 171-184.

OHNO S., TRUJILLO J. M., KAPLAN W. D., KINOSITA R., 1961. Nucleolus organizers in the causation of chromosomal anomalies in man. Lancet, 2, 123.

OLDS P. J., STERN S., BIGGERS J. D., 1973. Chemical estimates of the RNA and DNA contents of the early mouse embryo. J. exp. Zool., 186, 39-46.

REAMER G. R., 1963. The quantify and distribution of nucleic acids in the early cleavage stages of the mouse embryo. Ph. D. Thes., Boston Univ.

SALPETER M. M., BACHMANN L., 1972. Autoradiography, 221-278. In HAYAT M. A., Principes and techniques of electron microscopy. Biological applications. Vol. 2, Van Nostrand Reinhold Co., New York.

SCHWARZACHER H. G., MIKELSAAR A. V., SCHNEDL W., 1978. The nature of the Ag-staining of nucleolus organizer regions : Electron and light-microscopic studies on human cells in interphase, mitosis and meiosis. Cytogenet. Cell Genet., 20, 24-39.

SCHWARZACHER H. G., MIKELSAAR A. V., SCHNEDL W., 1977. Silver staining of nucleolus organizer regions in man : light and electron microscopic observation, 45-50. In DE LA CHAPELLE A., SORSA M. Elsevier, North Holland Biomedical Press, Amsterdam.

STAHL A., LUCIANI J. M., DEVICTOR M., CAPODANO A. M., GAGNE R., 1975. Constitutive heterochromatin and micronucleoli in the human oocyte at the diplotene stage. Humangenetik, 26, 315-327.

STAHL A., MIRRE G., HARTUNG M., KNIBIEHLER B., NAVARRO A., $1977 . \quad$ Localization, structure and activity of ribosomal cistrons in the mouse oocyte during meiotic prophase I. In DE LA CHAPELlE A., SORSA M. Elsevier/North Holland Biomedical Press, Amsterdam, The Netherlands.

STAHL A., MIRRE C., HARTUNG M., KNIBIEHLER B., NAVARRO A., $1978 . \quad$ Localization and structure of nucleolar organizers in the oocyte during meiotic prophase. 1. Ann. Biol. anim. Bioch. Biophys., 18, 399-408.

STERN H., HOTTA Y., 1974. DNA metabolism during pachytene in relation to crossing over. Genetic, 78, 227-235.

TRES L. L., 1975. Nucleolar RNA synthesis of meiotic prophase spermatocytes in the human testis. Chromosoma, 53, 141-151.

WASSARMAN P. M., LETOURNEAU G. E., 1976. RNA synthesis in fully grown mouse oocytes. Nature (Lond.), 261, 73-74.

WOLGEMUTH-JARASHOW D. J., JAGIELLO G. M., HENDERSON A. S., 1977. The localization of rDNA in small, nucleolus-like structures in human diplotene oocyte nuclei. Hum. Genet., 36, 63-68.

WOLGEMUTH D. J., JAGIELLO G. M., HENDERSON A. S., 1979. Quantitation of ribosomal RNA genes in fetal human oocyte nuclei using rRNA : DNA hybridization in situ. Evidence for increased multiplicity. Exp. Cell Res., 118, 181-190. 